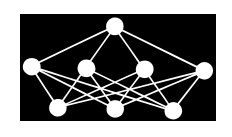

\title{
NEURAL NETWORK WORLD: OPTIMIZED SPIRAL SPHERICAL SOM (OSS-SOM)
}

\author{
Timotej Jagric, Matjaz Zunko
}

\begin{abstract}
The border effect is one of the problems, which can appear in the application of self-organizing maps (SOM). Different solutions were presented in the literature, but each of them has its drawbacks. In this paper we present a new method for overcoming the border effect - optimized spiral spherical SOM. We also show that standard measure of irregularity is not appropriate and present a modified version - Gaussian measure of irregularity. Our simulations suggest that the new variant of SOM achieves extremely low values of irregularity in comparison to other methods. At the end of the paper we present a software solution for the proposed method.
\end{abstract}

Key words: Self-organizing map, spherical SOM, border effect, topology

Received: March 19, 2013

Revised and accepted: October 24, 2013

\section{Introduction}

The self-organizing map was developed by Kohonen [10]. Original SOM uses unsupervised learning and is mainly applied in the area of clustering, pattern recognition, brain theory, biological modeling mathematics, signal processing, data mining [16]. The method clusters data and simultaneously preserves the topology of the input space. In the learning phase the data is divided into groups and is from the input space projected on the low-dimensional space. Neurons are positioned on a grid, which can be one-, two-, or high-dimensional. Mapping is achieved by a process of competition among neurons in the network [20]. Different types of SOM's, methods of learning, and possible application areas are described in Kohonen [10].

In applications researchers mostly use SOM which is based on a rectangular or hexagonal network topology [10]. However, these types of topologies suffer from the border effect, since at the border they have a restricted neighborhood [20]. As further explained in Schmidt [21] lower number of neighbors reduces the capacity

\footnotetext{
*Timotej Jagric

Department for Finance, Faculty of Economics and Business, University of Maribor, Razlagova 14, 2000 Maribor, Slovenia. Tel.:+386 22290 343, timotej.jagric@uni-mb.si

$\dagger$ Matjaz Zunko

Department for Economic policy, Faculty of Economics and Business, University of Maribor, Razlagova 14, 2000 Maribor, Slovenia. Tel.:+386 22290 290, matjaz.zunko@uni-mb.si
} 
of bordering neurons to interact with other neurons during the learning process. In order to avoid the border effect, one has to use a regular topology. This means that every neuron has the same number of neighbors throughout the whole learning process [21].

The border effect can be avoided by placing the grid of neurons on a threedimensional object. Two possible objects were proposed as perfect candidates in the literature [21]: sphere and torus. Li et al. [12] showed a solution for the toroidal SOM. The main problem in SOM's which are built on torus is the visualization of results. Maps generated from a torus are difficult to interpret [8, 29].

Due to the difficulties mentioned above, Ritter [16] suggests that a curved topology may be better suited. A sphere can also be better visualized. One major shortcoming of a spherical model is that options of perfectly regular placement of neurons on the sphere are rather limited. These options are known as platonic solids $[16,7]$. Other arrangements of neurons on the sphere result in an irregular topology [21].

First suggestion on generating spherical lattice was given by Ritter [16] and is based on the idea of subdividing the sides of the icosahedron. Since in this way there is no flexibility in the size of the network other methods were developed $[17,7,28,13]$. The main drawback of these methods is the irregularity of the topology.

In this paper we address the problem of irregular topology in connection with the flexible network size. We build on idea provided in Jagric [9] and propose a method, which will guarantee a topology with highly regular topology and also allows a totally exible network size. As far as we know, such type of solution was not yet proposed in SOM literature. Additionally, we use a new variant of a measure of irregularity as described in Jagric [9], which will be used to determine the degree to which irregularity affects the SOM. Finally, we provide a software implementation of the methods described in the paper.

The paper is organized as follows. First we explain the relation between the size of the neural network and the border effect. In section three we show a new method for measuring regularity of the network topology. In section four we propose a new variant of the spherical SOM - optimized spiral spherical SOM. In section five we provide a short description of the software implementation of the method. Finally, we give concluding remarks in section six.

\section{Size of the network and the border effect}

The size of a network is essential in the process of model development. The selection of an appropriate size of a network depends on: the size of the data used in the learning phase, the structure of the data (number of variables) and application area. Additionally, one has also to take into account the type of the neural network which will be used in the application. Previous research has not provided any clear guidance on this subject $[3,21]$.

The network size of a classical SOM is flexible and should depend on the size of the input space [10]. Vesanto [24] suggests the network size to be as big as possible, however, he also remarks on the computational intensity. In our experiments we have clearly faced the trade-off between bigger size and the computational intensity. 
Jagric T., Zunko M.: Neural network world: Optimized spiral spherical...

In his SOM toolbox Vesanto [24] suggests for the initial network size formula $5 \sqrt{n}$ where $n$ is the number of observations. However, this rule cannot be applied in all problems and does not refer to any SOM topology specifically neither do other suggestions in the literature (e.g. Wendel and Buttenfield [26], Brereton [1]) therefore the eligibility of any guidance for the spherical SOM is to be examined.

In this paper we have no intention to discuss the appropriate size of the network. The above description should only make clear that the size is a relevant factor when using neural networks, and therefore the solutions to the border effect mentions in the introduction, should allow flexible size of the network.

Classical flat SOM has no problem with flexibility in the size of the network $[11,24]$. In spherical SOM the flexibility in the size of the network depends on a method, which was used to distribute the neurons on a sphere. In method proposed by Ritter [16], neurons are arranged by subdividing an icosahedron recursively. This means that one can only have specific number of neurons in a network. The size by Ritter's rule is defined as [21]:

$$
N=2+10 \cdot 4^{n}, n=0,1,2, \ldots
$$
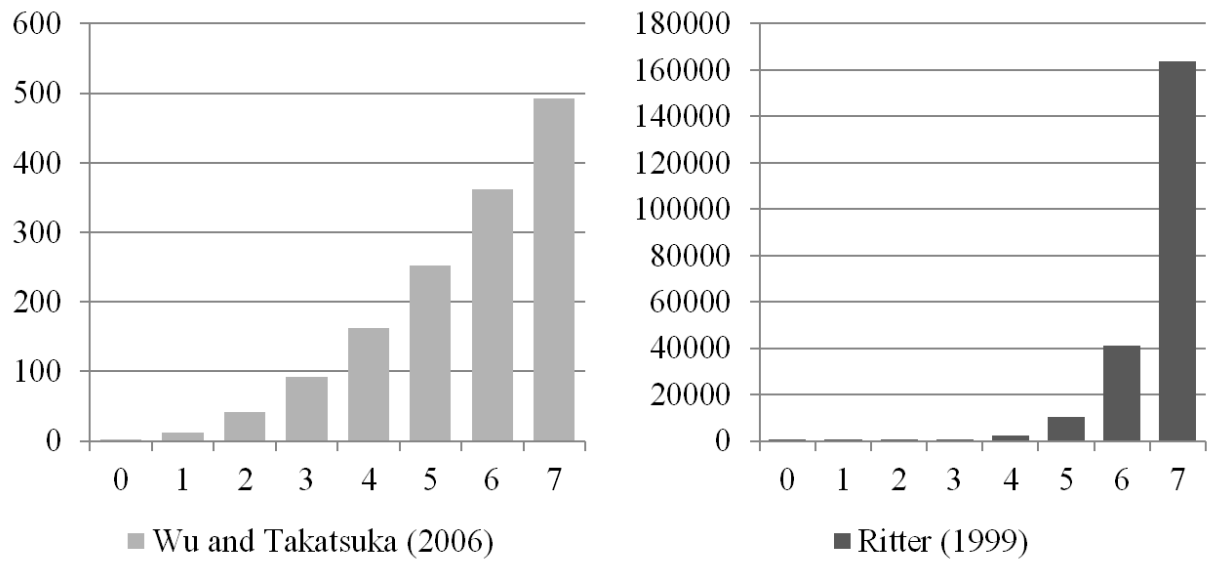

Fig. 1 Different tessellation methods and possible network size (example for up to 7 iterations). Note: X-axis - number of divisions/iterations, Y-axis - number of neurons. Source: Authors calculations.

Possible sizes of networks, which are constructed according to Ritter's [16] method are presented in Figure 1. Wu and Takatsuka [29] have modified the above algorithm. They redivide the original icosahedron in each iteration. The size of the network is therefore a bit more flexible and is defined as [21]:

$$
N=2+10 \cdot n^{2}, n=0,1,2, \ldots
$$

Other solutions are more flexible. Rakhmanov et al. [15] proposed another method which is extremely flexible in neuron number but for which, Wu and Takat- 
suka [29] showed that fails to satisfy the irregularity conditions [21]. We will come back to their idea of generalized spiral set, later extended by Saff and Kuijlaars [17], and upgrade it further in our section 4. Nishio et al. [13] introduced a method based on helix, which is divided in any number of parts. The method provides quite good distribution of neurons, but the main problems remain in both poles of the sphere where the starting and the ending points of the helix are placed. Other solutions also exist as shown in Schmidt [21], but none of them provides a satisfying results.

\section{Measuring the border effect}

In order to avoid any border effect a topology must satisfy two conditions [21]: first, each neuron should cover the same quantity of space on the sphere, and second, each neuron should have the same number of neurons in neighborhood.

The spherical SOM was developed by Ritter [16]. Even though it does not have borders, as this is the case in plane topologies, there is still a problem of uniformly arranging arbitrary number of neurons. Irregularity of the network is the equality of the number of neighborhood neurons when observing all the neurons in the map for different sizes of neighborhood area. Therefore, completely regular map has the same number of neighborhood neurons for each of the neurons [14].

In Fig. 2 we give an example of the neighborhood on a spherical SOM, which was generated according to generalized spiral set. We show the example for the case where we select neuron $\eta=800$ and neighborhood of a size $r=0.8$.

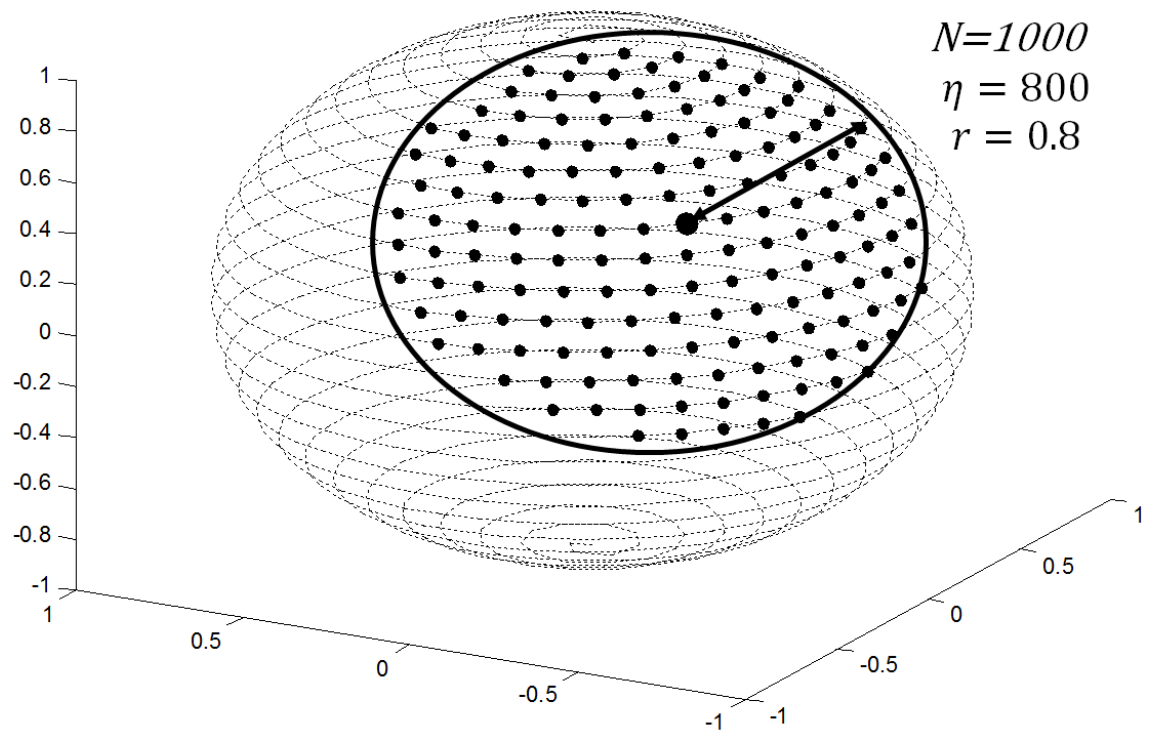

Fig. 2 Neighborhood on a spherical SOM. Source: Authors calculations. 
Jagric T., Zunko M.: Neural network world: Optimized spiral spherical...

Nishio et al. [14] introduced the concept of uniformity as the variance of the number of neighborhood neurons at specific radius $r$ over all neurons $\eta$ on the sphere. It is defined by Equation 3, where $f(\eta, r)$ is the numbers of neurons within radius $r$ from neuron $\eta[14]$ :

$$
V(r)=\frac{n \sum_{\eta} f(\eta, r)^{2}-\left(\sum_{\eta} f(\eta, r)\right)^{2}}{n^{2}} .
$$

From the definition of variance $V(r)$ it follows that it can only be a real positive number or zero, the latter is achieved in the ideal case when the number of neighborhood neurons $f(\eta, r)$ is the same for all neuron $\eta$. Since this definition of uniformity depends on the neighborhood size parameter $r$, Nishio et al. [14] proposed a general measure of irregularity (originally called UNTIDINESS by Nishio et al. [14]), which take into account various neighborhood sizes $r$ [14]:

$$
\text { IRREGULARITY }=\int_{\theta=0}^{\theta=\pi / 2} V\left(2 \sin \left(\frac{\theta}{2}\right)\right) d \theta .
$$

This measure integrates values of uniformity for all neighborhood sizes from 0 to $\sqrt{2}$, as follows from the observation that $2 \sin (\theta / 2)$ is the length of a chord for center angle $\theta$, but authors did not specify an argument for the upper bound. Since IRREGULARITY integrates non-negative numbers, its value can be a real positive number or zero. Lower values are preferable. When the arrangement is completely regular, IRREGULARITY is zero.

When thinking about regularity of neuron arrangement on the sphere, two things should be considered [9]. First is that if we use Euclidean distances at spherical topology, we ignore the true three-dimensional placing of neurons on a curved surface. We constrain neurons to the surface of the sphere but if are using Euclidean distances we are measuring distances of the shortest line segments that are going through the sphere's interior. For instance the distance between antipodal points is the length of the line that goes through the center, the diameter. But the actual distance between these points according to constrained neurons is the distance we would cover if we would travel from one point to the other on the surface of the sphere. Instead of straight lines of Euclidean geometry it is preferable to use geodesics and therefore geodesic distances or great-circle distances. The simplest approach to calculate distances on a unit sphere is by the definition of the dot product for vectors $\mathbf{x}_{i}$ and $\mathbf{x}_{j}[2]$ :

$$
d=\arccos \left(\mathbf{x}_{i} \cdot \mathbf{x}_{j}\right) .
$$

This formula can have rounding error if the distance between points is small compared to circumference. In this case we can use the haversine formula [22] or the Vincenty's formulae [25]. For reasonable size of the network, the rounding error is rather small and can be ignored. If for example the optimized spiral spherical SOM network has a size of 2562 neurons (equivalent to 4 th level of recursive subdivision of the icosahedron), maximal difference between method based on Equation 5 and Vincenty's formulae is $3.11 \cdot 10^{-12}$. Nevertheless, we will use Equation 5 .

The second issue we should consider when thinking about regularity of neurons on the sphere is to take into account the learning algorithm of the spherical SOM 
[9]. During learning, the neurons which are close to each other will be activated. This will lead to adoption process, which moves selected neurons closer to the input $\mathbf{y}$. This will have a smoothing effect on the weight vectors of neighboring neurons. The continued learning results in global ordering. A very central role in the relaxation process has so-called neighborhood function, which is a smoothing kernel defined over the neurons [10].

The measure of uniformity by Nishio et al. [14] as variance of the number of neighborhood neurons is appropriate only if all the neurons in the neighborhood of the best matching unit would be updated with the same weight, which is true if we use the box neighborhood. Another widely applied and on our opinion more suitable is the use of the Gaussian function as the neighborhood function

$$
f_{t}\left(\mathbf{x}_{i}\right)=\exp ^{-\frac{d\left(\mathbf{x}_{B M U}, \mathbf{x}_{i}\right)^{2}}{2 \sigma^{2}(t)}},
$$

where $\sigma(t)$ is the learning rate parameter at time $t$ and $x_{B M U}$ is the best matching unit. This function provides smoother local relaxation as it operates with fade-out effect, as described by [10].

For this neighborhood function the irregularity measure written above could not be appropriate any more. The neurons are updated according to the distance from the best matching unit and so the irregularity measure should take this distance into account. As proposed in [9] we will next examine the idea of the measure that uses the Gaussian function at learning process. Let the total learning update be the sum of the neighborhood function for neuron $\eta$ at learning parameter $r$

$$
g(\eta, r)=\sum_{i=1}^{N} \exp ^{-\frac{d\left(\mathbf{x}_{\eta}, \mathbf{x}_{i}\right)^{2}}{2 r^{2}}} .
$$

It does not present the actual total learning update as during learning process this amount is then multiplied with learning rate function at time $t$ before applied to current weights. But these multiplications only scale total learning update according to the current step in the learning process so for the research of the regularity of the neuron arrangement, we can neglect them.

After computing the total learning update $g(\eta, r)$ at learning parameter $r$ for all neurons, we can define uniformity as the variance of them

$$
V(r)_{\text {Gaussian }}=\sum_{i=1}^{N} \frac{(g(i, r)-\overline{g(i, r)})^{2}}{N} .
$$

When the neurons are ideally uniformly arranged the total learning update is the same for all neurons and consequently the variance is zero. When the arrangement of neurons is not uniform the total learning update differs from neuron to neuron and the variance is positive.

This irregularity measure is subject to learning parameter $r$ so we propose an independent measure of irregularity, in the same manner as Nishio et al. [14], that is as the integral of uniformity from minimum learning parameter $r=0$, to the largest sensible learning parameter $r=\max _{i, j} d(i, j)(r=\pi$ at unit sphere). At this learning parameter, the fade-out effect at the antipodal points is rather small 
Jagric T., Zunko M.: Neural network world: Optimized spiral spherical...

so most of the neurons on the sphere are updated with the same weight and the spherical SOM would hardly order according to the data. The actual minimum and maximum learning parameter $r$ in the learning process should be chosen carefully and are subject to number of neurons and the data, and there is not a uniform recipe for them (as with Kohonen's [10] SOM). But they should not go outside the sensible borders written above and that is the reason we choose them

$$
\text { IRREGULARITY } Y_{\text {Gaussian }}=\int_{r=0}^{\pi} V(r)_{\text {Gaussian }} d r .
$$

\section{Optimized spiral spherical SOM - a new model of spherical SOM}

Starting point of the idea presented in Jagric [9] is to use generalized spiral set introduced by Rakhmanov et al. [15] as initial distribution of neurons on a sphere. There are two main reasons for this decision:

- Generalized spiral set algorithm is a fast and efficient way of distributing any number of neurons on a sphere.

- The distribution gives a satisfying regularity of the topology, especially at lower number of neurons.

The method of generalized spiral set was motivated by hexagonal tilings and numerical experimentation [17]. Rakhmanov et al. [15] have developed an algorithm, which uses spherical coordinates $(\theta, \phi), 0 \leq \theta \leq \pi, 0 \leq \phi \leq 2 \pi$. The coordinates are defined as [17]:

$$
\begin{gathered}
\theta_{k}=\arccos \left(h_{k}\right), h_{k}=-1+\frac{2(k-1)}{N-1}, 1 \leq k \leq N, \\
\phi_{k}=\left(\phi_{k-1}+\frac{3.6}{\sqrt{N}} \frac{1}{\sqrt{1-h_{k}^{2}}}\right)(\bmod 2 \pi), 2 \leq k \leq N-1, \phi_{1}=\phi_{N}=0 .
\end{gathered}
$$

The coordinates are then used to define a set of points $\widehat{\omega}_{N}=\left\{\theta_{k}, \phi_{k}\right\}_{k=1}^{N}$, which author name a generalized spiral set on $\left.S^{2}[17,15]\right)$. The process of calculating the positions of points on a sphere can be easily geometrically interpreted. In the first step, we divide the sphere with $N$ horizontal planes. The planes have predefined distance between each other. The distance is set to $2 /(N-1)$ units. In this way we generate $N$ circles on the sphere. Due to the properties of the sphere are the first and the last circles of a size of a point. Using geographical terms one could name them north and south poles. The algorithm places on each circle only one point. After the point is placed on the selected circle, we move up to next circle. The points $\left(\theta_{k}, \phi_{k}\right)$ are positioned counterclockwise on circles with fixed distance, which is independent of $k$ [17]. The process is graphically demonstrated in Figure 3 where an example for $N=6$ is given. 


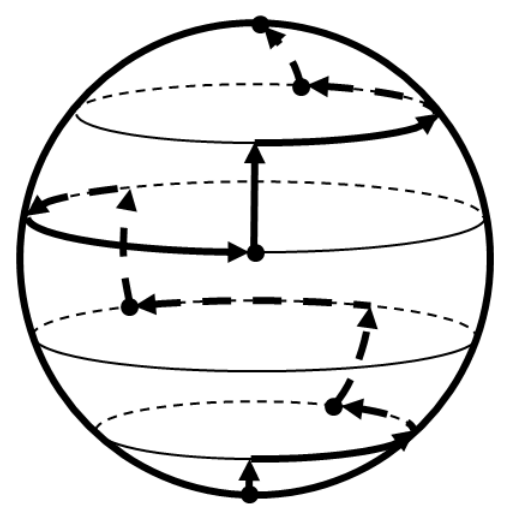

Fig. 3 Construction process of generalized spiral points for $n=6$. Source: E. B. Saff and A. B. J. Kuijlaars [17], pp. 5-11.

The distance between points is a fixed value. In the algorithm above, the distance is defined as $\left(\phi_{k}-\phi_{k-1}\right) \sqrt{1-h_{k}^{2}}$. The value is calculated based on the result of Habicht and van der Waerden [6]. They suggest that it should be approximately [17]:

$$
\delta_{N}=\left(\frac{8 \pi}{\sqrt{3}}\right)^{1 / 2} \frac{1}{\sqrt{N}} \approx 3.809 \frac{1}{\sqrt{N}}
$$

that is

$$
\left(\phi_{k}-\phi_{k-1}\right) \sqrt{1-h_{k}^{2}}=3.809 \frac{1}{\sqrt{N}} .
$$

As we can see, the definition of the distance includes a constant. The value of the constant was defined on numerical simulation. No analytical solution has yet been provided for defining the value of the constant [17]. In Figure 4 we give numerical experimentation in order to show the relationship between the selected value of the constant, size of the sphere, and different types of irregularity measures. Our results confirm that the value cannot be set fix for different sizes, however there exists an interval for best solutions (see Figure 4).

In order to have more general definition Saff and Kuijlaars [17] introduced a parameter $C$ in place of the constant. The parameter $C$ can be adjusted appropriately for the application at hand:

$$
\begin{gathered}
\theta_{k}=\arccos \left(h_{k}\right), h_{k}=-1+\frac{2(k-1)}{N-1}, 1 \leq k \leq N, \\
\phi_{k}=\left(\phi_{k-1}+\frac{C}{\sqrt{N}} \frac{1}{\sqrt{1-h_{k}^{2}}}\right)(\bmod 2 \pi), 2 \leq k \leq N-1, \phi_{1}=\phi_{N}=0 .
\end{gathered}
$$



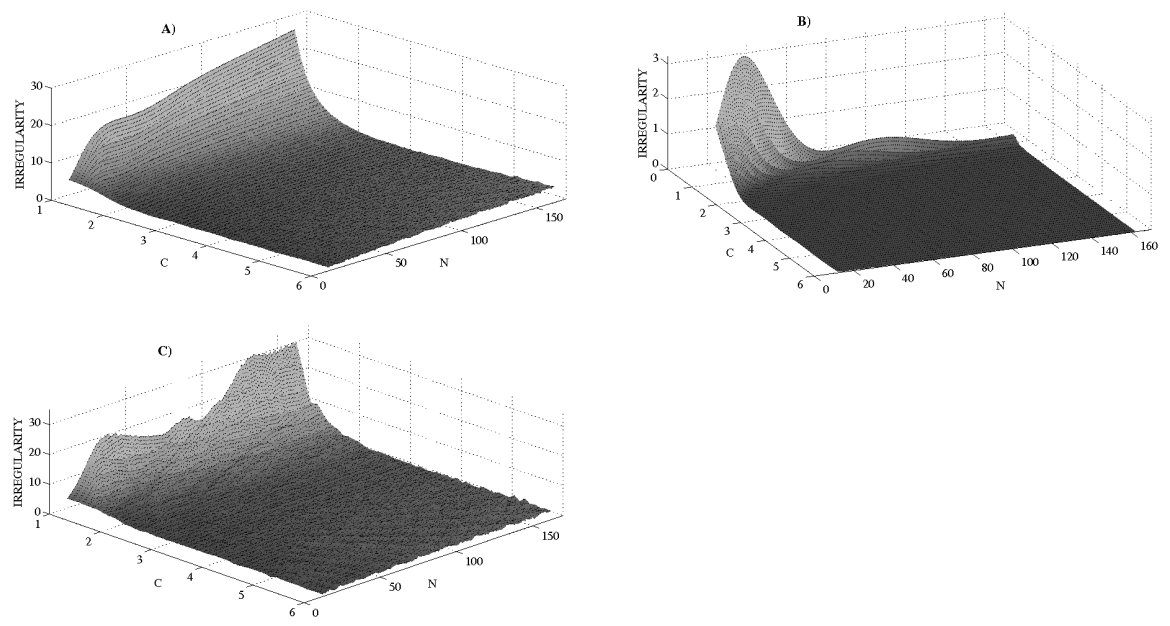

Fig. 4 Relationship between $C, N$ and different measures of irregularity - numerical simulation. Note: A) Irregularity defined by Nishio et. al [14], B) Gaussian measure of irregularity, $C$ ) Irregularity as maximum variance; $N$ - size of the network, $C$-selected value of the constant. Source: Authors calculations.

As we can see, the proposed algorithm has some drawbacks:

- Different treatment of points on a sphere. First and the last point (the poles) are fixed.

- No analytical solution to the definition of the distance between points on the sphere.

- It also turns out that the algorithm does not distribute points on the sphere optimally according to the measure of irregularity. The distribution can be improved.

The idea in Jagric [9] is to optimize the generalized spiral points with a method based on Fekete points. Fekete points on the sphere are the points that minimize (dimensionless) Coulomb's energy if we treat points as equal charged particles [17]:

$$
E=\sum_{1 \leq i<j \leq N}\left|\mathbf{x}_{i}-\mathbf{x}_{j}\right|^{-1} .
$$

This is also known as the Thomson problem and there are several algorithms for solving it for an arbitrary $N$ (Monte Carlo, genetic algorithms, relaxation, local relaxation). Claxton and Benson [4] proposed an approach that is based on the mechanical interpretation of the behavior of a system of particles that interact according to forces derived from Coulomb's law. They described an iterative procedure, based on the method of steepest descent, to displace the particles towards a 
minimum energy configuration [4]. The Coulomb's force acting on the $i$-th particle is $[5]$ :

$$
\overrightarrow{F_{i}}=\sum_{j=1}^{N} \frac{\overrightarrow{\mathbf{x}}_{i}-\overrightarrow{\mathbf{x}}_{j}}{\left|\overrightarrow{\mathbf{x}}_{i}-\overrightarrow{\mathbf{x}}_{j}\right|^{3}} \quad(i \neq j)
$$

Iteratively, each particle is allowed to move in the direction of the resultant force (17) acting on it. As this will generally take the particle off the sphere, it must be re-normalized by the distance from the center [4]:

$$
\overrightarrow{\mathbf{x}}_{i}(n e w)=\frac{\overrightarrow{\mathbf{x}}_{i}+\gamma \vec{F}_{i}}{\left|\overrightarrow{\mathbf{x}}_{i}+\gamma \vec{F}_{i}\right|},
$$

where $\gamma$ is a scale factor which determines the extent of the displacement. Assignment of an optimum value for it is difficult as it depends on the size of the sphere and the number of particles. Claxton and Benson [4] stated that a reasonable compromise can be obtained by setting

$$
\gamma=\frac{K}{\max _{i=1, \ldots, N} \vec{F}_{i}}
$$

where $K$ is a constant equal to 0.1 or 0.2 .

This force relaxation algorithm seeks to balance forces rather than attempting to minimize the energy directly and leads univocally to a solution that in general is only a local minimum [4]. In our opinion, it is reasonable to start with analytically derived points distribution that is known as a uniform distribution (such as generalized spiral points) and then optimizing that distribution with force relaxation algorithm. If we start with generalized spiral points then this algorithm will on general move only points around the poles as there appear inconsistencies due to the spiral turns. The points around the equator will in general maintain their positions. This is good for visualization of the spherical SOM as spiral points distribution is nice to visualize with Delaunay triangulation.

Since Thomson problem and spherical neuron distribution with the lowest irregularity are only related problems and not the same, we performed some experiments to check whether any modifications in force relaxation algorithm better fit our goal. We tried several modifications in the definition of the force and different constants $K$ while monitoring Coulomb's energy of particles and the irregularity of so arranged neurons. Our empirical results showed that although it leads to higher Coulomb's energy, better results on irregularity is given by the force relaxation algorithm with the force:

$$
\vec{F}_{i}=\sum_{j=1}^{N} \frac{\overrightarrow{\mathbf{x}}_{i}-\overrightarrow{\mathbf{x}}_{j}}{\left|\overrightarrow{\mathbf{x}}_{i}-\overrightarrow{\mathbf{x}}_{j}\right|^{1}} \quad(i \neq j)
$$


Jagric T., Zunko M.: Neural network world: Optimized spiral spherical...

Instead of raising the length of the resultant between two particles in normalization to the third power we normalize with the length only. It turns out to be the best to keep the constant $K$ equal 0.2 for all iterations in the algorithm. This approach lowers Coulomb's energy at every iteration but it does not necessarily lower irregularity at every iteration. For instance at $N=42$, we got results presented at Fig. 5.

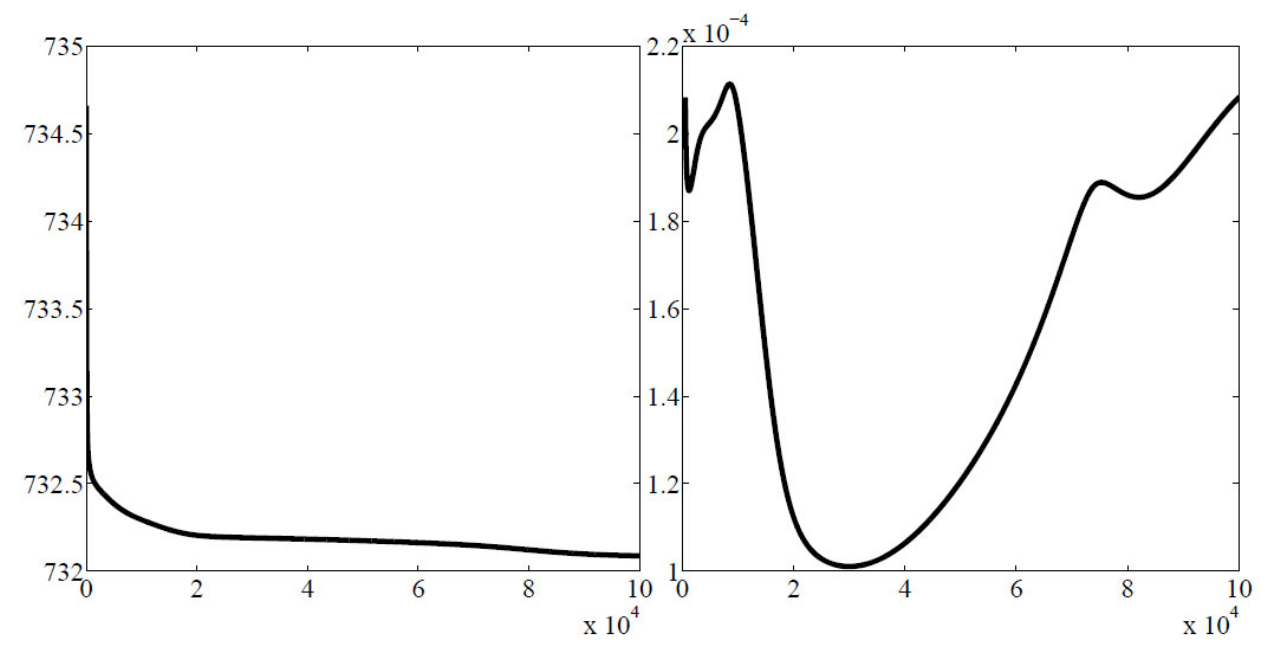

Fig. 5 Optimizing general spiral set $(N=42)$. Note: Left graph - Coulomb's energy. Right graph - IRREGULARITY. X-axis - number of iterations. Source: Authors calculations.

It is clear that although modified force relaxation algorithm moves particles to better and better energy distribution, we do not have a control about optimality of irregularity during iterations. In the first few hundred iterations it lowers in large amount but we are not able to know at which iteration it will reach its minimum. Because of that we added to the algorithm a function of monitoring the irregularity at every iteration, executed it for chosen number of iterations and then taking as the best neuron distribution the one where the minimum was reached.

For example at $N=42$ it can be seen on Figure 5 that we iterate 100000 times and then took as the best the arrangement at around 30000-th step. At $N=2562$ we iterated 11000 times (which lasted for around 60 hours) and still did not reach any local minimum of irregularity (see Figure 6).

For practical purposes it is enough to iterate only for around 1000 times as in this first iterations the irregularity lowers at most and the algorithm does not last for long. Further iterations are for reaching high precisions only.

Figure 7 presents the results we achieve with our algorithm. We iterate for 1000 times starting from generalized spiral points arrangement and then took the one with the lowest irregularity. The difference is obvious. Based on these results we can be fairly confident that we have excluded the border effect from SOM completely. 


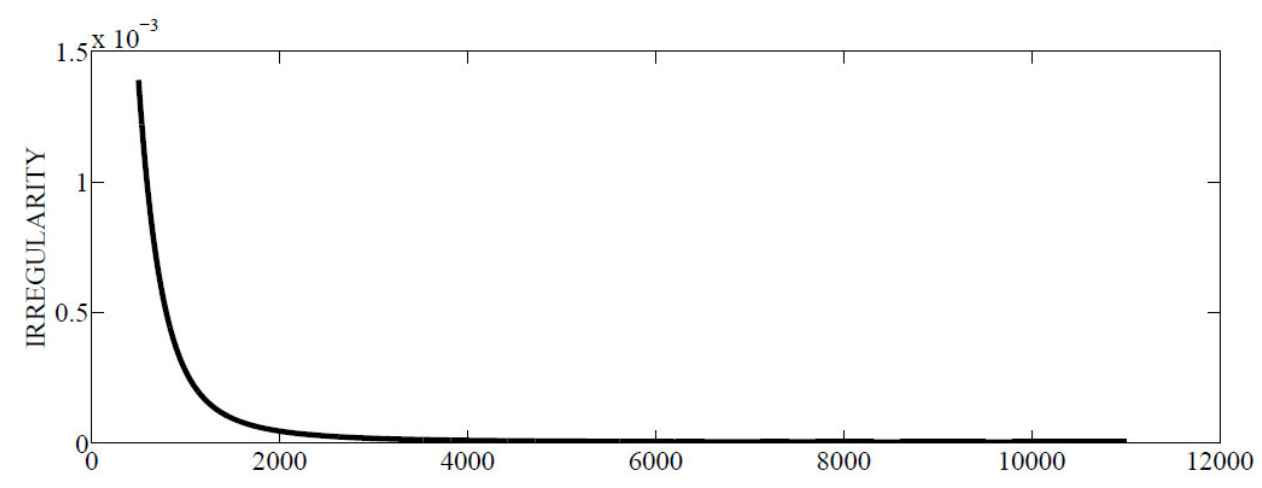

Fig. 6 Optimizing general spiral set $(N=2562)$. Note: X-axis - number of iterations. Y-axis - Gaussian measure of irregularity. Source: Authors calculations.

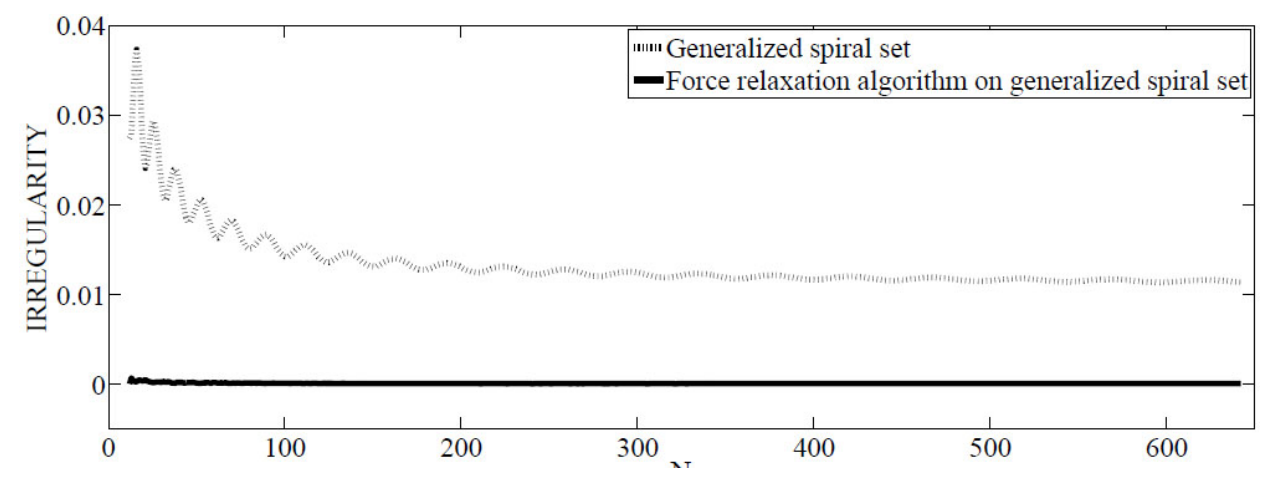

Fig. 7 Difference in Gaussian measure of irregularity between SOM based on generalized spiral set and optimized spiral spherical SOM. Note: X-axis - number of iterations. Y-axis - Gaussian measure of irregularity. Source: Authors calculations.

\section{Software implementation}

After we have eliminated the border effect for an arbitrary size of the network with optimized spiral spherical SOM we now have a powerful tool for applied research. We have implemented it into an application with user friendly graphical interface. Application is conducted in Matlab and enables a variety of SOM characteristics. The user can choose among several starting neuron arrangements and different training settings (screenshot on Figure 8).

In the first GUI, which is used for setting model parameters, user can choose the size of the network, type of the neighborhood function and model of BMU. Special section is devoted to the selection of method for generating the spherical SOM. User can select between random distribution, helix, generalized spiral set 
Jagric T., Zunko M.: Neural network world: Optimized spiral spherical...

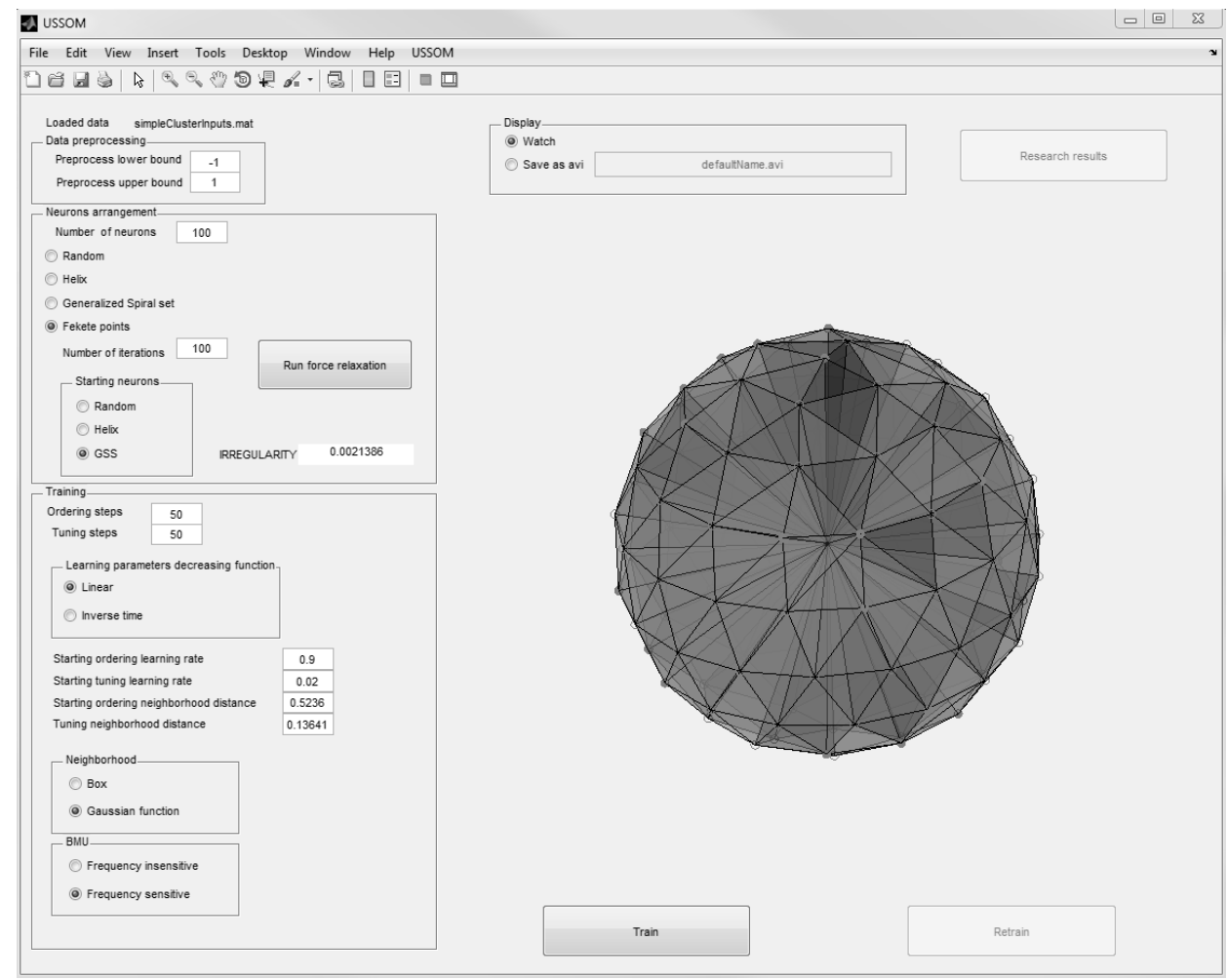

Fig. 8 Software implementation of the optimized spiral spherical SOM - GUI for model parameters.

and optimized spiral based on different initial distributions. The GUI is also used to train the network. Spherical SOM plotting is based on Delaunay triangulation and the triangles are colored according to the scale of surfaces of all triangles. All results are graphically presented and can be saved also in video file.

Training results can be investigated thoroughly (screenshot on Fig. 9). We have developed a separate GUI for analyzing the training result. This allows the user to plot the irregularity measure, glyph (sphere is scaled into mountains and valleys and colored accordingly so the clusters are more evident - method was introduced by Sangole [18]), neurons arrangement, sample hits, weight for each input variable, and subgroups of samples. For better visualization, the sphere is displayed from both sides: front and back.

\section{Conclusion}

Self-Organizing Map (SOM) was introduced by Kohonen [10]. Due to its ability to handle high dimensional data, SOM is mostly applied in the area of clustering, classification, and data mining. In most application a SOM based on a two-dimensional 


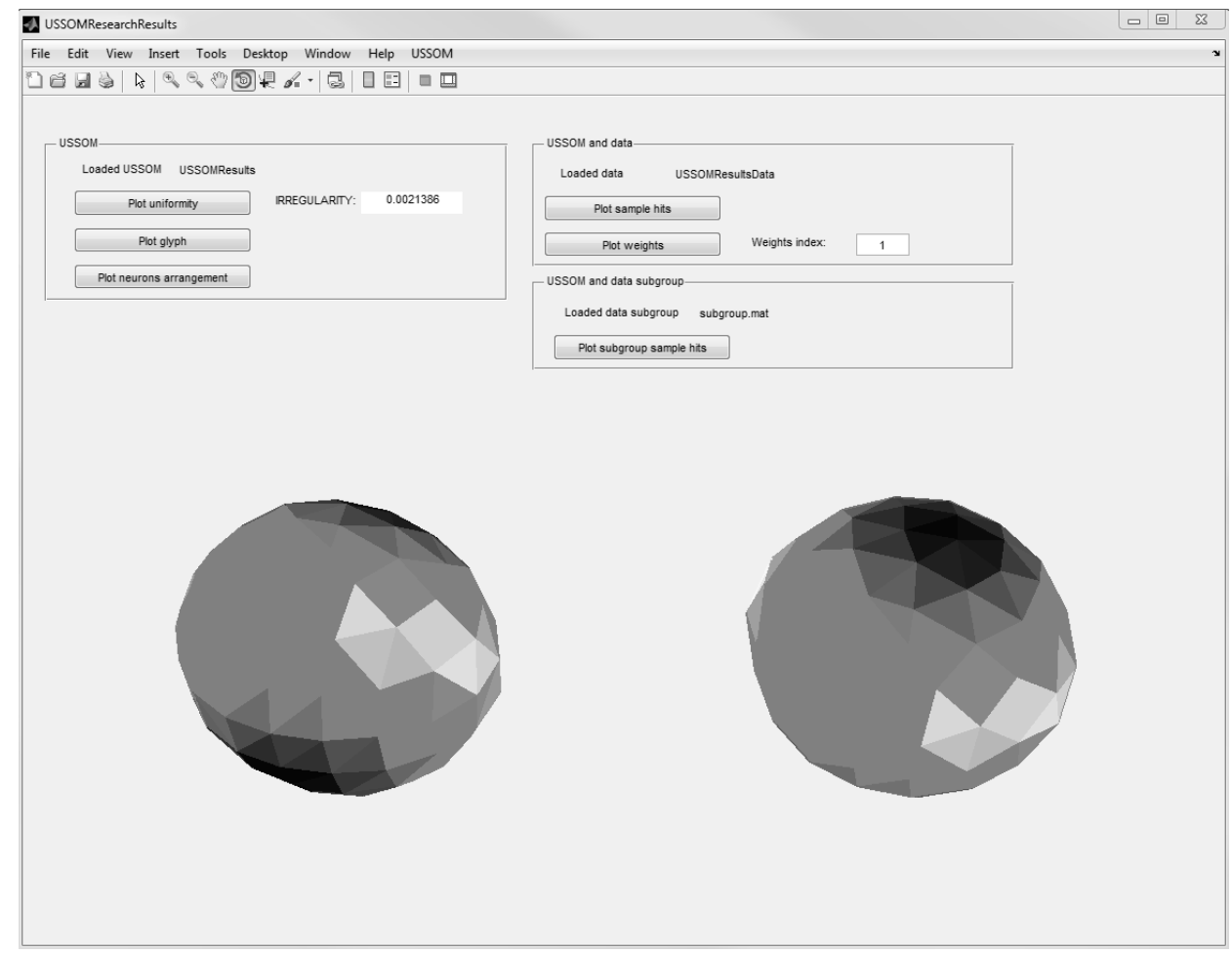

Fig. 9 Software implementation of the optimized spiral spherical SOM - GUI for analyzing training results.

rectangular or hexagonal grid is applied. But, as it was already noticed by Kohonen [10], such networks may suffer from the border effect.

When the network is learning, the information is passed from the winning neuron to its neighbors. In a two-dimensional rectangular or hexagonal grid the neurons on the edge or close to the edge of the grid have fewer neighbors as neurons in the center. Therefore, the learning process has different impact on individual neurons in the grid, which is called the border effect. To overcome this problem, different solutions were developed in the past. Some of them are based on spherical SOMs.

In this paper we further tested the idea, which was introduced in Jagric [9], and propose a new version of a spherical SOM - optimized spiral spherical SOM. Such SOM has a flexible network size and also guarantees the best possible level of irregularity. Additionally, we also propose a modified version of the irregularity measure, Gaussian measure of irregularity. We have developed a software solution, which integrates the proposed method and gives the possibility to compare the modifications with some other known types of spherical SOM. 
Jagric T., Zunko M.: Neural network world: Optimized spiral spherical...

\section{Acknowledgement}

The paper "Optimzed Spiral Spherical SOM (OSS-SOM)" was produced within the framework of the operation entitled "Centre for Open Innovation and Research of the University of Maribor". The operation is co-funded by the European Regional Development Fund and conducted within the framework of the Operational Programme for Strengthening Regional Development Potentials for the period 2007 - 2013, development priority 1: "Competitiveness of companies and research excellence", priority axis 1.1: "Encouraging competitive potential of enterprises and research excellence".

\section{References}

[1] Brereton: Self organising maps for visualising and modelling. Chemistry Central Journal, 6(Suppl 2):S1, 2012.

[2] Bronstein, I. N., Semendjajew, K. A. and Musiol G., and H. Mühlig: Taschenbuch der Mathematik. Verlag Harri Deutsch, Thun, expanded edition, 2001.

[3] Cho, S., Jang, M., and Reggia, J. A.: Effects of varying parameters on properties of selforganizing feature maps. Neural Processing Letters, V4(1), 1996, pp. 53-59. Available from: http://dx.doi.org/10.1007/BF00454846.

[4] Claxton, T.A. and Benson, G.C.: Stereochemistry and Seven Coordination. Canadian Journal of Chemistry, 44, 1966, pp. 157-63.

[5] Erber, T., and Hockney, G.M.: Equilibrium configurations of $\mathrm{N}$ equal charges on a sphere. J. Phys. A: Math. Gen., 24, 1991, pp. 1369-1377.

[6] Habicht W. and B. L. van der Waerden: Lagerung von Punkten auf der Kugel, Math. Ann. 123, 1951, pp. 223-234.

[7] Harris, J. M., Hirst, J. L., and Mossinghoff, M. J.: Combinatorics and graph theory. Springer, New York, 2000.

[8] Ito, M., Miyoshi, T., and Masuyama, H.: The characteristics of the torus self organizing map. In Proceedings of 6th International Conference ON Soft Computing (IIZUKA2000), volume A-7-2, 2000, pp. 239-244, Iizuka, Fukuoka, Japan. Available from: http://mylab.ike.tottoriu.ac.jp/ mijosxi/act1997-/\#Oral.

[9] Jagric, T.: Kako izboljsati merjenje bancne integracije v evrskem obmocju [How to improve measuring financial integration in the Euro area]. Bancni vestnik, 62, 2013, pp. 36-40.

[10] Kohonen, T.: Self-Organizing Maps. Springer, 3rd edition. 2001.

[11] Kohonen, T., Hynninen, J., Kangas, J., and Laaksonen, J.: Som pak: The self-organizing map program package. Technical Report A31, Helsinki University of Technology, Laboratory of Computer and Information Science, FIN-02150 Espoo, Finland, 1996.

[12] Li, X., Gasteiger, J., and Zupan, J.: On the topology distortion in self-organizing feature maps. Biological Cybernetics, V70(2), 1993, pp. 189-198. Available from: http://dx.doi.org/10.1007/BF00200832.

[13] Nishio, H., Altaf-Ul-Amin, M., Kurokawa, K., and Kanaya, S.: Spherical SOM and arrangement of neurons using helix on sphere. IPSJ Digital Courier, 2, 2006, pp. 133-137.

[14] Nishio, H., Md. Altaf-Ul-Amin, Kurokawa, K., Minato, K., and Kanaya, S.: Spherical SOM with arbitrary number of neurons and measure of suitability. WSOM 2005, 2005, Paris.

[15] Rakhmanov, E. A., Saff, E. B., and Zhou, Y. M.: Minimal discrete energy on the sphere. Mathematical Research Letters, 1, 1994, pp. 647-662.

[16] Ritter, H.: Self-organizing maps on non-euclidean spaces. In Oja, E. \& Kaski, S., editor, Kohonen Maps, 1999, pages 97-110. Elsevier, Amsterdam. Available from: citeseer.ist.psu.edu/ritter99selforganizing.html. 
[17] Saff, E.B., and Kuijlaars, A.B.J.: Distributing Many Points on a Sphere. The mathematical intelligencer, 19(1), 1997, pp. 5-11.

[18] Sangole, A. P.: Data-driven modeling and visualization using spherical self-organizing feature maps, Doctor of Philosophy (PhD) Thesis, 2003. Department of Mechanical and Materials Engineering. Faculty of Engineering. The University of Western Ontario, London, Ontario, Canada.

[19] Sangole, A. and Knopf, G. K.: Visualization of randomly ordered numeric data sets using spherical self-organizing feature maps. Computers \& Graphics, 27(6), 2003, pp. 963-976. Available from: http://www.sciencedirect.com/science/article/B6TYG-49S75953/2/0913af2271d2f83a833452eea7ebddf9.

[20] Sangole, A.P., and Leontitsis, A.: Spherical Self-Organizing Feature Map: An Introductory Review. International Journal of Bifurcation and Chaos, 16(11), 2006, pp. 3195-3206.

[21] Schmidt, C.R.: Effects of Irregular Topology in Spherical Self-Organizing Maps. A Thesis Presented to the Faculty of San Diego State University, 2008.

[22] Sinnott, R.W.: Virtues of the Haversine, Sky and Telescope 68(2), 1984, pp. 159.

[23] Skupin, A. and Agarwal, P.: Introduction: What is a self-organizing map? In Agarwal, P. and Skupin, A., editors, Self-Organising Maps: Applications in Geographic Information Science. Wiley, 2008.

[24] Vesanto, J.: Som toolbox: implementation of the algorithm, 2005. Available from: http://www.cis.hut.fi/projects/somtoolbox/documentation/somalg.shtml

[25] Vincenty, T.: Direct and Inverse Solutions of Geodesics on the Ellipsoid with Application of Nested Equations, Survey Review, 23(176), 1975, pp. 88-93.

[26] Wendel, J. and Buttenfield, B. P.: Formalizing Guidelines for Building Self-Organizing Maps. GIScience, Short Paper Proceedings, Zurich, Switzerland, September 2010.

[27] Wasserman, S. and Faust, K.: Social network analysis: methods and applications. Cambridge University Press, Cambridge; New York, 1994.

[28] Wu, Y. and Takatsuka, M.: Geodesic self-organizing map. In Erbacher, R. F., Roberts, J. C., Grohn, M. T., and Borner, K., editors, Proc. SPIE Vol. 5669, volume 5669 of Visualization and Data Analysis 2005, pp. 21-30. SPIE, 2005.

[29] Wu, Y. and Takatsuka, M.: Spherical self-organizing map using efcient indexed geodesic data structure. Neural Networks, 19(6-7), 2006, pp. 900-910. Available from: http://www.sciencedirect.com/science/article/B6T08-4K719S6-2/2/f6d26607f815781a5bd3c fe5df5d6214. 